\title{
REFLEXIONES SOBRE EL REFERÉNDUM: ¿UNA SENCILLA HERRAMIENTA EN DEMOCRACIA?
}

\author{
Rosa María Fernández RiveirA \\ Departamento de Derecho Constitucional \\ Facultad de Derecho \\ Universidad Complutense de Madrid \\ ferrosa@ucm.es
}

\begin{abstract}
RESUMEN
Se exponen una serie de consideraciones sobre el referéndum como instrumento democrático por excelencia, para poner de relieve sus elementos e implicaciones desde todos los puntos de vista.

Palabras clave: democracia, Estado de Derecho, Constitución, consulta, referéndum.

\section{ABSTRACT}

We hereby offer a number of reflections on the Referendum as the ultimate democratic tool, in order to make emphasis on its bits and bobs taking into account the different points of view to be looked at.
\end{abstract}

Keywords: Democracy, Rule of Law, Constitution, Consultation, Referendum.

\section{ZUSSAMENFASSUNG}

Es werden eine Reihe von Überlegungen zum Referendum als einem demokratischen Instrument «par excellence» erörtert, um seine Elemente und Auswirkungen unter allen Gesichtspunkten berauszustellen.

Schlüsselwörter: Demokratie, Rechtsstaat, Verfassung, Volksbefragung, Referendum.

SUMARIO: I. INTRODUCCIÓN.-II. LA ENORME IMPORTANCIA Y PROTAGONISMO QUE HA ALCANZADO EL REFERÉNDUM EN LA SOCIEDAD ACTUAL DEL SIGLO XXI.-III. EL NUEVO HÁBITAT DEL REFERÉNDUM: NUEVAS TECNOLOGÍAS, FAKE NEWS, CAMPAÑAS ELECTORALES EN CLAVE INTERNACIONAL, MICRO Y MACRO FINANCIACIÓN DEL PROCESO, TRANSPARENCIA, GLOBALIDAD...-IV. ¿POR QUÉ ES IMPORTANTE CONOCER PREVIA Y CLARAMENTE LOS EFECTOS Y CONSECUENCIAS QUE TENDRÁ EL REFERÉNDUM?-V. ¿CABEN TODO TIPO DE PREGUNTAS/CONSULTAS EN ESTA HERRAMIEN- 
TA PARTICIPATIVA?-VI. ¿QUÉ PUEDE SIGNIFICAR DESATENDER EL REFERÉNDUM, EN TANTO QUE NO ACTUAR VINCULADO A SU RESPUESTA?-VII. ¿QUIÉN TIENE O DEBERÍA TENER LA COMPETENCIA PARA CONVOCAR UN REFERÉNDUM?-VIII. CONCLUSIONES.

In Memoriam, profesor y compañero José Antonio Alonso de Antonio...

Escribo estas breves reflexiones con mucho cariño para un compañero de «profesión», una persona sencilla y cercana que dedicó gran parte de su vida y trabajo a la Universidad.

\section{INTRODUCCIÓN}

Las ideas que se exponen en estas páginas tienen como protagonista una herramienta de democracia directa, el referéndum. Pero más bien tienen como objetivo romper una lanza en favor de la categoría «herramienta» en busca de nueva regulación normativa y redimensión en pleno siglo XxI. Y ello en el ánimo de poner en evidencia tantos recientes y no tan recientes intentos, en la historia del referéndum, que tratan de sobredimensionar la naturaleza de una simple herramienta político-jurídica para encontrar en ella responsabilidades y culpabilidades a problemas de muy diversa índole. O lo que es todavía peor, hallar soluciones mágicas para escenarios heterogéneos y complejos. La crisis de la democracia representativa, el nuevo marco global, transnacional e internacional del poder político, el tsunami tecnológico que afecta cualquier diseño institucional y funcional del Estado, son realidades complejas que requieren de soluciones complejas.

Vivimos tiempos en los que, de manera inesperada, se ha dado ingente protagonismo al referéndum, y es probable que ello haya propiciado la tentación de «matar al mensajero», que no es más que eso, un vehículo de transmisión de la voz de la soberanía popular, un micrófono que se acerca al «pueblo» para escucharle. Pero en ningún caso, este mecanismo de democracia directa ha sido, o es, la solución de un problema político, ni la causa de una circunstancia, de una realidad, injusticia o descontento. Un buen diseño del referéndum será imprescindible para conocer la opinión del pueblo a él convocado. Unas buenas «reglas del juego», en tanto que normas reguladoras del mismo, devienen básicas para el óptimo logro del objetivo. Pero no esperemos más, no queramos más, estamos tan solo frente a una «herramienta». O quizá debamos preguntarnos, ¿realmente estamos tan solo ante una sencilla «herramienta»? 
Los motivos por los que se han convocado y celebrado referéndums responden a lógicas muy heterogéneas, casi en algunos casos contradictorias. Su incremento cuantitativo choca frontalmente con su anodina y obsoleta consideración/comprensión política y su cuasi inexistente regulación jurídica. Pero solo una lógica pueril atribuiría tal escalada cuantitativa a una «fe desmedida en la democracia representativa» ${ }^{1}$.

El 23 de junio de 2016 tenía lugar en el Reino Unido el referéndum ${ }^{2}$ que preguntaba al pueblo británico acerca de su salida o permanencia en la Unión Europea y de un total de 33.577.342 votantes registrados votaron a favor de apoyar el Brexit, 17.410.742, y votaron permanecer en Europa, 16.141.241. Dos años después, este complejo proceso sigue en transición y tiene al gobierno británico profundamente dividido sobre la conveniencia o no de asumir una salida de Europa y, en su caso, sobre cómo hacerlo. También se ha publicado, concretamente en julio de 2018, un interesante documento: Report of the independent Commission on Referendums, acerca de las luces y sombras de esta herramienta en democracia ${ }^{3}$. En él hay muchas reflexiones que tratan de esclarecer la esencia del referéndum

${ }^{1}$ J. SAn Vicente Feduchi, «Referéndum», Eunomía. Revista de cultura de la legalidad, núm. 14 (2018), p. 278: «El referéndum ha sido en ocasiones duramente criticado por los defensores de la participación por ser, esencialmente, el método de toma de decisiones mayoritario más extremo [...] por su riesgo de simplificar una decisión compleja en una respuesta binaria y de dinamitar la posibilidad de consenso [...]. Inicialmente los referéndums fueron justificados como una capa más en el sistema de checks and balances que impediría a las elites sacar provecho de su poder. Muchos han criticado que el referéndum ofrece la esperanza espuria de la elección popular, cuando en realidad son herramientas excelentes para que el Gobierno y los grupos de presión más influyentes adquieran legitimidad para una política determinada o una institución».

2 J. M. Castella Andreú, «El referéndum sobre el Brexit: una historia inacabada», Revista de Derecho Político, núm. 97 (2016), p. 302: «El lugar que ocupa el referéndum en la democracia representativa británica, en la que el referéndum es un instrumento extraño, aunque no por ello no utilizado, sobre todo en los últimos años, en relación con decisiones políticas de orden constitucional. Así, el referéndum pugna con la tradición de soberanía parlamentaria y ello explica su tardía regulación, su contestación política y doctrinal, su carácter consultivo y su uso excepcional. Pero a pesar de lo anterior, el referéndum ha encontrado su lugar en el sistema constitucional, sobre todo en los últimos años, como instrumento en manos del primer ministro y la mayoría, lo que propicia su convocatoria con base en factores políticos que dependen de su conveniencia política, sobre todo durante los gobiernos Cameron desde 2010. Se traslada al cuerpo electoral una decisión fundamental en lugar de ser tomada por las instituciones representativas».

${ }^{3}$ La autoría de este excelente documento se atribuye al The Constitution Unit (bttps:// constitution-unit.com), que dirigen los académicos Meg Russell, profesora del British and Comparative Politics del UCL y directora de The Constitution Unit, y Alan Renwick, subdirector de The Constitution Unit y director de investigación de Independent Commission on Referendums. Vid. Report of the Independent Commission on Referendums, julio de 2018. 
y, al mismo tiempo, generar un «activo científico» que permita analizar si el proceso vivido en Reino Unido en 2016 fue suficientemente transparente y oportuno como para asumir abiertamente el resultado arrojado entonces.

Por traer a colación otros escenarios (salvando muchas diferencias), el 1 de octubre de 2017 Cataluña vive un intento de «jornada de referéndum» en el que se pregunta al pueblo de Cataluña si quiere ser un Estado independiente en forma de república. Una jornada complicada con claros incumplimientos de las «reglas del juego» en materia de referéndum tanto en la Constitución española de 1978 (art. 92), como en la Ley Orgánica 2/1980, de 18 de enero, sobre regulación de las distintas modalidades de referéndum.

El 25 de mayo de 2018 se votaba en Irlanda rechazar la octava enmienda de su Constitución que desde 1983 prohíbe el aborto. El 66,4 por 100 votó a favor frente al 33,6 por 100 que votó en contra, siendo la cifra de participación de un 64,51 por 100 de la población con derecho a voto.

La lista de ejemplos podría ser mucho más larga: la independencia de Escocia (2014), la independencia de Quebec en Canadá (1980 y 1995), los sucesivos referéndums de ratificación de un texto constitucional para Europa (2004), para cerrar el conflicto bélico con las FARC en Colombia (2016), etc. La pretensión de estas breves páginas no es analizar la figura del referéndum - muchos autores en la doctrina ${ }^{4}$ lo han hecho de manera pormenorizada-, sino más bien contribuir a un debate sobre uno de los múltiples aspectos que suscita esta herramienta mal dimensionada y usualmente utilizada en escenarios de enorme complejidad. Una herramienta de democracia directa o semidirecta que añade o sustrae ciertas dosis de «legitimidad», que recurre a la soberanía popu-

${ }^{4}$ N. Pérez Sola, La regulación constitucional del referéndum, Jaén, Universidad de Jaén, 1994; A. L. Alonso De Antonio, «La ley catalana de consultas populares de 2014», Revista de Derecho Político de la UNED, núm. 101 (2018), pp. 607-628; C. RodrígueZ-Aguilera DE PRAT, «El uso del referéndum en la España democrática (1976-1986)», Revista de Estudios Políticos, núm. 75 (1992), pp. 131-164; J. L. LóPEZ GONZÁLEZ, El referéndum en el sistema español de participación política, Valencia, Universidad Politécnica de Valencia, 2005; I. Lasagabaster HerRarte, Consulta o referéndum. La necesidad de una nueva reflexión jurídica sobre la idea de democracia, Bilbao, Lete, 2008; M. R. RiPOLlÉS SERRANO, «Notas acerca de la ley orgánica sobre distintas modalidades de referéndum», en AAVV, Comentarios a la Constitución española de 1978, Valencia, Universidad de Valencia, 1980; E. Linde PaniAGUA, «Art. 92. Referéndum», en Ó. Alzaga VillaAmil (dir.), Comentarios a las Constitución española de 1978, t. VII, Madrid, Edersa-Cortes Generales, 1998; M. Menéndez Alzamora, Participación y representación politica, Valencia, Tirant lo Blanch, 2009; J. Oliver Araujo, «El referéndum en el sistema constitucional español», Revista de Derecho Político, núm. 29 (1989), pp. 115-184, y P. CRUZ VilLALÓN, «El referéndum consultivo como modelo de racionalización constitucional», Revista de Estudios Políticos, núm. 13 (1980), pp. 145-168. 
lar para la búsqueda de decisiones y la incentivación de la participación. En definitiva, un gran potencial escondido en escenarios de cambios $^{5}$ y que pide a gritos nuevos enfoques, más reflexión y nuevas pautas normativas regulatorias.

El Tribunal Constitucional en España en una vieja sentencia (STC 63/1987, de 20 de mayo, FJ 5. ${ }^{\circ}$ ) señalaba: «La participación directa que en los asuntos públicos ha de corresponder a los ciudadanos es la que se alcanza a través de las consultas populares previstas en la propia Constitución (arts. 92, 149.1.32, 150.1, 152.2, 167.3 y 168.3), procedimientos estos en los que habrán de hacerse presentes, sin duda, los partidos y, en general, las agrupaciones políticas, mas no como titulares del derecho mismo a la participación, sino, en lo que a los partidos políticos se refiere, como instrumentos fundamentales que son para hacerla posible, concurriendo, como la Constitución quiere, a la formación y manifestación de la voluntad popular». Finalmente, los hábitos democráticos son imprescindibles en el órgano convocante. El Gobierno adquiere en este sentido una peculiar responsabilidad en el funcionamiento del instrumento referendario. Con estas ideas nuestro máximo intérprete constitucional perfilaba los límites y «ropajes jurídicos» de una compleja figura.

Como cualquier herramienta, el referéndum comporta diversas partes estructurales: su heterogénea naturaleza como herramienta de participación directa; su contenido indefinido y amplio, revelador del «sentir» del pueblo soberano; su regulación o «reglas del juego» (jurídicas, políticas, económicas, sociales, culturales...), y sus consecuencias o efectos. Estos últimos, a pesar de moverse en una oscilación dual aparentemente simple, esto es, vinculatoriedad o no, producen una marea ingente de «otros» efectos a veces difíciles de racionalizar en una teoría argumentativa jurídico-política al uso, tales como inclinaciones de las cifras macroeconómicas, oscilaciones de las tendencias en las relaciones internacionales, narrativas en la opinión pública de «gran visceralidad», etc. Recordemos cómo el 4 de noviembre de 2016, solo cinco meses después del referéndum sobre el Brexit, la prensa escrita británica tildó de «enemies of the people» a los jueces de la High $\operatorname{Court}^{6}$, un episodio sin precedentes en este país, en el que la institución de la judicatura siempre ha disfrutado de un profundo respeto y reconocimiento.

5 A. Garrorena Morales, Escritos sobre la democracia: la democracia y la crisis de la democracia representativa, Madrid, Centro de Estudios Políticos y Constitucionales, 2015.

6 Daily Mail, 4 de noviembre de 2016: «Fury over, "out of touch". Judges who defied $17,4 m$ Brexit voters and could trigger constitutional crisis. ENEMIES OF THE PEOPLE». 
Las líneas que se suceden, siendo consciente quien las escribe del descrito escenario y su enorme complejidad, solo pretenden poner de relieve algunas ideas sobre el referéndum, reivindicando en todo momento su esencia «instrumental». Tales sugerencias son: $a$ ) la enorme importancia y protagonismo que ha alcanzado el referéndum en la sociedad actual del siglo xxI; b) el nuevo «hábitat» del referéndum (nuevas tecnologías, fake news, campañas electorales en clave internacional, micro y macro financiación del proceso, transparencia, globalidad...); c) ¿por qué es importante conocer previa y claramente los «efectos y consecuencias» que tendrá el referéndum?; d) ¿caben todo tipo de preguntas/consultas en esta herramienta participativa?; $e$ ) ¿qué puede significar desatender el referéndum en tanto que no actuar vinculado a su respuesta?, y $f$ ¿ ¿quién tiene o debe tener la competencia para convocar un referéndum?

\section{LA ENORME IMPORTANCIA Y PROTAGONISMO QUE HA ALCANZADO EL REFERÉNDUM EN LA SOCIEDAD ACTUAL DEL SIGLO XXI}

La sociedad actual refleja el enorme protagonismo que ha adquirido esta herramienta de participación directa. Se data por los expertos que tal vez el primer referéndum nacional en la sociedad moderna puede fecharse en la Francia de 1793 que ratificaba con sufragio universal un texto constitucional que nunca llegaría a aplicarse. Pero los datos ponen de relieve que los referéndums oscilan, en forma y fondo, de unos países a otros y de unos contextos históricos a otros. Actualizados a 2017, podemos ver que desde 1990 Italia ha celebrado 56 referéndums nacionales; Irlanda, 27; Eslovenia, 25; Lituania, 21; Eslovaquia, 18; Nueva Zelanda, 14; Uruguay, 12; Botsuana, 11; Polonia, 10; Latvia, 10; Hungría, 9; Islandia, 8; Rumania, 7; Dinamarca, 7; Bulgaria, 5; Luxemburgo, 4; Estonia, 4; Portugal, Malta, Francia y Australia, 3; Reino Unido, Suiza, España y Austria, 2; Noruega, Grecia, Finlandia, República Checa, Chipre, Costa Rica y Canada, 1. Y no han celebrado ninguno durante estos años países como Estados Unidos, Namibia, Japón, Jamaica, Israel, Alemania, Chile o Bélgica. Frente a todos estos países, Suiza ha celebrado 261 referéndums federales ${ }^{7}$.

Los cambios en la frecuencia de recurrir a esta herramienta de unos países a otros reflejan también los momentos de mayor o menor intensi-

7 SUDD 2017, «Datenbank und Suchmaschine für Direkte Demokratie» (página web). 
dad en la agenda y actividad política del país. Así, por ejemplo, el repunte de un gran volumen de referéndums vividos en torno a 1990 en diversos países del mundo estuvo provocado por un fenómeno de «constitutional making» como consecuencia del escenario de erradicación de la antigua Unión Soviética y del final de la denominada «guerra fría», produciéndose el alumbramiento de nuevos países y sus correspondientes regímenes. Las expectativas sobre la democracia han cambiado ${ }^{8}$. En los primeros años tras la Segunda Guerra Mundial, se fraguó una concepción minimalista sobre la participación y la democracia, que tan solo ofrecía a los votantes votar cada legislatura a sus representantes, pero años después el concepto de «democracia» y de «participación» ha ido reformándose. Quizá las expectativas ciudadanas de poder tomar parte en las decisiones públicas son distintas y el escenario de la globalidad y de las nuevas tecnologías ha propiciado, entre otros muchos factores, tales cambios. El referéndum es una herramienta con gran potencial participativo, pero con un diseño bastante antiguo y con una legislación bastante vetusta en muchos países de nuestro entorno. Podemos poner de relieve que el apoyo dado a esta herramienta por la sociedad de diversos países ha cambiado sustancialmente en los últimos años. La conclusión es que los ciudadanos del siglo XXI están ciertamente divididos, unos demandan más frecuencia en su uso y cambios en su regulación (Taiwán, Venezuela, Finlandia, Australia, Suiza, Hungría, Israel, Eslovaquia, República Checa y Eslovenia), y, por el contrario, otros consideran menos útil y necesaria esta herramienta que hace diez años (España, Estados Unidos, Reino Unido, Francia, Austria, Rusia, Dinamarca, Japón, Austria y Polonia, entre otros) ${ }^{9}$.

Pero si echamos una mirada a la permanente adaptación de nuestros procesos participativos (la clásica separación de poderes, la emergencia de nuevos poderes públicos, la «cierta» crisis de las instituciones públicas, las nuevas demandas sociales y, sobre todo, los temas sobre los que se han ido sucediendo los referéndums en los últimos años) podemos concluir que han adquirido una incontestable relevancia. Los principales asuntos sobre los que se han convocado son:

${ }^{8}$ R. J. Dalton y M. Gray, «Expanding the Electoral Marketplace», en B. E. Cain, R. J. Dalton y S. Scarrow (eds.), Democracy Transformed? Expanding Political Opportunities in Advanced Industrial Democracies, Oxford, Oxford University Press, 2003, pp. 21-40.

9 ISSP Research Group, International Social Survey Programme Citizenship-2004. Vid. también G. A. Almond y S. Verba, The Civic Culture Political Attitudes and Democracy in Five Nations, California, Newbury Park, 1963, pp. 310 y ss. 
1. Asuntos de la vida política ordinaria tales como, la conformidad o no con una determinada ley que se ha aprobado por el Parlamento en una determinada legislatura o incluso, sobre un asunto que aún no se ha aprobado por el órgano legislativo, pero que cuenta con un detallado proyecto. Por ejemplo, en 1996 en Polonia el Parlamento sometió cuatro preguntas a los ciudadanos sobre ciertos aspectos de un programa de privatizaciones del Gobierno ${ }^{10}$ que se articulaba parlamentariamente. En Islandia, el art. 26 de la Constitución, en cambio, permite referéndum si el presidente se opone a la firma de una determinada ley tramitada y aprobada en el Parlamento ${ }^{11}$.

2. Preguntas sobre cuestiones intimamente ligadas a la ética, la moral o la dimensión espiritual. Este grupo de procesos de participación directa suelen ser equivalentes a los contenidos de aquellas leyes que permiten a los grupos parlamentarios voto al margen de la disciplina de grupo o lo que también se ha denominado «voto en conciencia». Portugal ha celebrado dos referéndums sobre el aborto en 1998 y en 2007; Irlanda celebró consultas vía referéndum para aceptar o no el divorcio en 1986 y 1995 , para abolir la pena de muerte en 2001, sobre los matrimonios del mismo sexo en 2015 y sobre el aborto en 1983 (1992) y en 2018; en Italia se hicieron referéndums sobre el aborto en 1981, sobre el divorcio en 1974 y sobre embriones y técnicas de reproducción asistida en 2005.

3. Reformas o modificaciones del texto constitucional. Estas consultas son las más frecuentes y numerosas. En la mayoría de casos la propia Constitución regula en su articulado los supuestos de previsión de referéndum cuando esta va a ser reformada o cambiada (arts. 167-168 CE de 1978, 89 de la Constitución francesa ${ }^{12}, 140$ de la Federal Constitution of

10 http://articles.chicagotribune.com/1996-02-21/business/9602210297_1_turnout-priva tization-referendum.

${ }_{11}$ «If althing has passed a bill, it shall be submitted to the President of the Republic for confirmation not later than two weeks after it has been passed. Such confirmation gives it the force of law. If the President rejects a bill, it shall nevertheless become valid but shall, as soon as circumstances permit, be submitted to a vote by secret ballot of all those eligible to vote, for approval or rejection. The law shall become void if rejected, but otherwise retains its force» (bttps://www.constituteproject.org/constitution/Iceland_1999.pdf).

12 «Los referéndums en Francia están asociados a una mitología particular, a momentos críticos de la República. Un referéndum precipitó la dimisión de un presidente, el general De Gaulle en 1969, y otros llevaron a Francia al borde de la salida del proyecto europeo, como el de 1992 sobre el Tratado de Maastricht o el de 2005 sobre la Constitución de la UE. Otros toparon con un desinterés de la ciudadanía, como el de 2000, que redujo el mandato presidencial de siete a cinco años. El presidente Enmanuel Macron contempla un referéndum ante la posibilidad de que la oposición torpedee su proyecto de revisión constitucional, prioridad para el primer semestre de 2018» (El País, 4 de febrero de 2018). 
the Swiss Confederation de 1999, 88 de la Constitutional Act of Denmark de 1953, 46 de la Constitución de Irlanda de 1937, 128 de la Commonwealth of Australia Constitution Act de 1900, 138 de la Constitución italiana de 1947, etc.). Al mismo tiempo y de manera contraria, Portugal prohíbe cualquier referéndum sobre reformas o modificaciones constitucionales (art. 115, sec. 4.a), de la Constitución de 1976).

4. Asuntos relativos a la independencia o autodeterminación. Son el grupo minoritario dentro de esta herramienta de participación. Y así, a pesar de que es palmario el incremento de las consultas en los últimos años, por el contrario, sobre independencia o autodeterminación el número de consultas ha descendido sustantivamente con relación a etapas históricas pasadas marcadas por procesos tales como la «descolonización», el periodo de entreguerras mundiales, la caída del comunismo en los países del este o la emergencia de nuevos Estados tras conflictos bélicos o guerras civiles. Señala Qvortrup que, a pesar de la importancia del contexto o marco que ofrece argumentos para tal herramienta y a pesar del resultado de la misma, siguen siendo determinantes aspectos tales como: las «supermayorías» requeridas, la «forma» o redacción de la pregunta que configura la consulta, la limitación de a quién o quiénes les está permitido o prohibido votar en el referéndum, etcétera ${ }^{13}$.

5. Cuestiones de cesión de soberanía y participación en organizaciones internacionales. La participación en organizaciones internacionales, en tanto en cuanto constituye una cierta «cesión de soberanía», parece lógico que sea consultada al titular por excelencia de aquella. El pueblo y solo él debe decidir en quién, cómo y hasta cuánto «delega» su soberanía. Ahora bien, tal sencillez o linealidad de argumento ha adquirido una complejidad de ropajes sin parangón, como ponen de relieve, sobre todo, los múltiples referéndums sobre los tratados internacionales constitutivos de la Unión Europea (sobre el Tratado de Maastricht en Dinamarca, Irlanda y Francia en 1992 y otra vez en Dinamarca en 1993; sobre una Constitución para Europa en España, Francia, Holanda y Luxemburgo en 2005; sobre el Tratado de Lisboa en Portugal y en Irlanda en 2008 y 2009, etc.).

Paralelamente, también se celebraron referéndums para tomar parte en organizaciones internacionales como la OTAN (Eslovaquia y Hungría en 1997 y Eslovenia en 2003), para ratificar acuerdos de libre comercio (Costa

${ }_{13}$ M. QvorTRup, «Referendums on Independence, 1860-2011», The Political Quarterly, vol. 85, núm. 1 (2014), pp. 57-64. 
Rica en 2007) o para formar parte de la Corte Penal Internacional (Irlanda en 2001).

Muchos países requieren de referéndums para los casos referidos y solo una gran mayoría alcanzada en el seno del Parlamento, sede de la soberanía popular, podría evitar tal decisión de someter la cuestión a consulta popular. Así, por ejemplo, la Constitución de Dinamarca de 1953 (art. 20) requiere cinco sextos de mayoría parlamentaria para sortear el referéndum en casos de cesión de soberanía por pertenencia a organismo internacional, o, según reza la Constitución francesa de 1958 (art. 88.5), tres quintos en ambas Cámaras.

\section{EL NUEVO HÁBITAT DEL REFERÉNDUM: NUEVAS TECNOLOGÍAS, FAKE NEWS, CAMPAÑAS ELECTORALES EN CLAVE INTERNACIONAL, MICRO Y MACRO FINANCIACIÓN DEL PROCESO, TRANSPARENCIA, GLOBALIDAD...}

Es evidente que en pleno siglo XXI el escenario ha cambiado. Vivimos en la sociedad tecnológica, en el mundo de las comunicaciones en red, y su lenguaje y fondo se han adaptado. Hay muchos ejemplos que así lo constatan. Varios autores han escrito acerca del papel «sin precedentes» que desempeñaron Google y Facebook en los días previos al referéndum del 25 de mayo en Irlanda sobre la legalización del aborto ${ }^{14}$.

El marco social en el que las campañas previas a cualquier referéndum se desarrollan, al margen de sus normas reguladoras (que son determinantes sin duda), preocupa cada vez más. Desinformación ${ }^{15}$, anuncios y publicidad de todo tipo claramente «sesgada» ${ }^{16}$, la enorme dificultad de paliar o mitigar la profunda influencia de la política exterior, la profunda caren-

${ }^{14}$ L. Carolan, «Responding to the Challenges of Digital Democracy during Ireland's Abortion Referendum», 7 de diciembre de 2018, disponible en www.constitution-unit.com.

${ }^{15}$ «Russian twitter accounts shared to the public, contributed relatively little to the overall Brexit conversation, russian news content was not widely shared among Twitter users, an only a tiny portion of the YouTube content was of a clear russian origin», V. NARAYANAN, M. Elswah, B. Kollanyi y P. Howard, «Russian Involvement and Junk News during Brexit», Computational Propaganda Research Project, Data Memo núm. 2017.10, Oxford, 2018.

16 «Facebook has launched further transparency measures, including a searchable archive of US-election related advertisements on Facebook and Instagram. These measures are still in development and so it is too early to tell how effective they will be», E. GraHAM-HarRIsON, «As Polls Narrow before the Abortion Vote. Is Rural Ireland Setting up a Brexit Moment?», The Observer, 20 de mayo de 2018. 
cia de transparencia, la complejidad para identificar a los actores materiales en la campaña y un largo etcétera complican y enturbian tal marco ${ }^{17}$.

\section{IV. ¿POR QUÉ ES IMPORTANTE CONOCER PREVIA Y CLARAMENTE LOS EFECTOS Y CONSECUENCIAS QUE TENDRÁ EL REFERÉNDUM?}

$\mathrm{Si}$, por un lado, es aceptado unánimemente el rol que desempeña el referéndum en las democracias contemporáneas ataviado de los principios/esencia de la democracia y orientado a poner voz a la voluntad popular, ha de quedar claro, a sensu contrario, el coste que tiene desoír o desatender el resultado del mismo.

Es cierto que existen normas reguladoras que configuran referéndums «no vinculantes», pero no es menos cierto que incluso en estos casos la opinión vertida por el titular de la soberanía popular produce un efecto tangible y concreto.

Expertos como Leduc establecen que en las culturas democráticas sólidas cualquier cambio importante sobre la esencia del Estado no puede ser hecho sin algún tipo de forma o consentimiento popular ${ }^{18}$. Si el referéndum puede dotar de legitimidad a una decisión mayoritaria, así como consolidarla otorgándola estabilidad y certeza, puede también debilitar un gobierno, crear conflicto en la sociedad y desorientarla ${ }^{19}$. Algunos autores como Dalton ${ }^{20}$ denominan tal fenómeno como el potencial «inhibidor de

17 D. Jahn y A. Storsved, «Legitimacy through Referendum? The Nearly Successful Domino-Strategy of the EU-Referendums in Austria, Finland, Sweden and Norway», West European politics, vol. 18, núm. 4 (1995), pp. 29 y ss.

${ }^{18}$ L. Leduc, The Politics of Direct Democracy: Referendums in a Global Perspectives, Toronto, University of Toronto Press, 2003, pp. 100-102.

19 Un problema inherente al referéndum se produce por las limitaciones que alberga este tipo de consulta «binaria». A diferencia de la gran mayoría de los asuntos políticos que requieren de diversos enfoques y de una gran variedad de opciones a ser consideradas en aras de llegar a una solución, el referéndum tiene que ceñirse a una dual respuesta limitadora, sin matices, ni añadidos, ni previos condicionamientos que podrían oscurecer o empobrecer el debate: «Such tribal divisions can persist long after the referendum itself, particularly if the margin of victory in the vote is narrow and those on the losing side feel inclined to continue their fight. This can have a longer-term inbibiting effect upon considered discussion of policy question. It can also harm bonds within society, leading to mutual suspicions and animosities», S. Chambers, «Constitutional Referendums and Deliberative Democracy», en M. Mendelsohm y A. Parkin (eds.), Referendum Democracy: Citizens, Elites and Deliberation in Referendum Campaigns, Basingstoke, Palgrave, 2001, pp. 230 y ss.

20 R. Dalton, Democratic Challenges, Democratic Choices: The Erosion of Political Support in Advanced Industrial Democracies, Oxford, Oxford University Press, 2004, pp. 28 y ss. 
una efectiva democracia», que a veces anida en el referéndum como tal o, de manera especial, en alguno de sus elementos: la campaña ${ }^{21}$ antes del día de la votación, el tipo y cualidad del debate, el resultado claro o «muy ajustado», el peso de los partidarios de una y otra opinión, etc.

Podríamos poner diversos ejemplos, pero tal vez el caso británico (referéndum sobre el Brexit 2016) ${ }^{22}$ ejemplifique mejor que ningún otro, por reciente, las reflexiones expresadas. Renwick señala que si el referéndum puede contribuir a la democracia (en su esencia y principios fundamentales, abundando u otorgando legitimidad a las decisiones políticas, ampliando el campo de elección y expresión de los votantes-ciudadanos, luchando por el apoyo público de las decisiones políticas, etc.) ${ }^{23}$, al mismo tiempo puede socavar o lesionarla. Y aquí debemos reflexionar: «Where a referendum takes place on a precisal proposal for change [...] it can make and legitimise a final decision. [...] where a referendum takes place on an imprecise proposal, difficulties can be created. As a consequence, parliament can find itself left with an instruction from voters but with wide disagreement on what that instruction means. That is particularly so if those who called for the change are not among those responsible for its implementation» ${ }^{24}$.

\section{V. ¿CABEN TODO TIPO DE PREGUNTAS/CONSULTAS EN ESTA HERRAMIENTA PARTICIPATIVA?}

¿Todo tipo de cuestiones o asuntos son susceptibles de ser sometidos a consulta de la ciudadanía mediante la celebración de un referéndum? Es posible que para responder a esta pregunta haya que formularse otras muchas preguntas y tratar de reflexionar en medio de otras tantas respuestas.

${ }^{21}$ En los procesos de referéndum, la campaña ejerce una influencia más fuerte que en las campañas de otros procesos electorales. Tal vez en estos casos las lealtades a partidos políticos preexistentes.

22 J. Sargeant, A. Renwick y M. Russell, «How Long Would it Take to Hold a Second Referendum on Brexit?», bttps://constitution-unit.com/2018/08/30/how-long-would-it-take-to-bolda-second-referendum-on-brexit/, e ÍD., «How could a second Brexit referendum be triggered?», https://constitution-unit.com/2018/09/07/bow-could-a-second-brexit-referendum-be-triggered/.

23 A. Renwick, The Politics of Electoral Reform: Changing Rules of Democracy, Cambridge, Cambridge University Press, 2010, pp. 175 y ss.

${ }_{24}$ Report of the Independent Commission on Referendums, London, The Constitution Unit UCL, 2018, pp. 62 y ss. 
La cuestión que se consulta debe ser concreta y clara, debe estar formulada de manera neutra, con una redacción que no incite a una respuesta determinada y con un lenguaje sencillo y accesible. En 2014 en Escocia tuvo lugar el referéndum sobre su independencia e inicialmente se consideró que la pregunta formulada podía incitar a cierta inclinación del electorado al sí: «Do you agree that Scotland should be an independent country?» La pregunta que finalmente fue sometida a consulta cambió la redacción por un texto más neutral: «Should Scotland be an independent country?».

Pero no solo hay que tener en cuenta aspectos formales, también debemos reflexionar sobre cierto discernimiento o limitación de contenidos. A veces la redacción resulta muy compleja o inapropiada porque el contenido también lo es. En 2016, la pregunta que el referéndum en Italia trasladó a sus ciudadanos fue: «¿Aprueba usted el texto de la ley constitucional referente a la "disposición para la superación del bicameralismo paritario, la reducción del número de parlamentarios, la limitación del coste de funcionamiento de la institución, la supresión del CNEL y la revisión del título V del capítulo II de la Constitución" aprobada por el Parlamento y publicada en la Gazzetta Ufficiale, núm. 88, del 15 de abril de 2016?» ${ }^{25}$.

¿Es apropiada la materia o asunto como objeto de referéndum? ¿Presenta tal asunto suficiente interés público como para que la participación sea elevada? ¿El referéndum es la vía o herramienta más apropiada para gestionar o decidir tal asunto o decisión? ¿Ha existido presencia o participación ciudadana previa en la materia objeto de referéndum, proporcionando el suficiente debate previo y/o reflexión? ¿Están claras las alternativas? ¿Es un referéndum multiopción ${ }^{26} \mathrm{o}$ es una alternativa entre una dualidad de soluciones? ¿Está claro para el legislador el potencial escenario consecuencia del resultado arrojado por el referéndum?

La Comisión de Venecia propone unas útiles recomendaciones sobre tales extremos en orden a validar la cuestión objeto de referéndum ${ }^{27}$. Pero en

25 Gazzetta Ufficiale, núm. 88, de 15 de abril de 2016.

${ }^{26}$ La mayoría de los escenarios de referéndums son binarios y solo existen excepcionalmente casos de multirrespuestas, como en 2013 en Jersey para elegir entre varias opciones sobre la composición de la legislatura y las diversas formas de elegir a sus representantes. Vid. https://www.bbc.co.uk/news/world-europe-jersey-21593531.

${ }_{27}$ Tales sugerencias son: unidad de forma, unidad de contenido y unidad de nivel jerárquico: «Unity of form: the same question must not combine a specifically-worded draft amendment with a generally-worded proposal or a question of principle; unity of content: except in the case of total revision of a text (Constitution, law), there must be an intrinsic connection between the various parts of each question put to the vote, in order to guarantee the free suffrage of the voter, who must not be called to accept or refuse as a whole provisions without an intrinsic link; the revision of several chapters of a text at the same time is equivalent to a total 
todo caso no establece límites claros en orden a evitar escenarios potenciales que no hacen sino desacreditar o lesionar la participación democrática.

Algunos países sí establecen asuntos o materias que no pueden, en ningún caso, ser objeto de referéndum: leyes sobre financiación, presupuestos e impuestos en Dinamarca, Italia, Países Bajos o Portugal [arts. 42(6), 75, sección 5, y 115(4) de sus textos constitucionales, respectivamente], o la legislación para ratificar tratados internacionales en Italia (art. 75 de la Constitución italiana de 1947). En otro sentido, textos constitucionales como el francés de 1958, reformado en 2008, explicitan sobre qué puede convocarse un referéndum (art. 11) y lo que debe interpretarse con carácter limitativo para todo aquello que no figure en tal precepto.

El caso español, regulado en la Ley Orgánica 2/1980, de 18 de enero, sobre las distintas modalidades de referéndum, señala en su art. 3: «El real decreto de convocatoria contendrá el texto íntegro del proyecto de disposición o, en su caso, de la decisión política objeto de la consulta». Como vemos, no limita ni positiva ni negativamente materias, lo que sí restringe es la posibilidad de convocarlo en determinadas circunstancias o periodos electorales concretos (art. 4).

\section{VI. ¿QUÉ PUEDE SIGNIFICAR DESATENDER EL REFERÉNDUM, EN TANTO QUE NO ACTUAR VINCULADO A SU RESPUESTA?}

Los ordenamientos jurídicos son los responsables de establecer cuándo un referéndum es vinculante y cuándo es meramente orientador o atiende a una demanda de legitimidad o de participación democrática. En ambos casos se producen efectos derivados del resultado de la consulta. Pero la gran diferencia estriba en que, mientras en el primer caso los pasos sucesivos a seguir por el legislador o el ejecutivo están «predeterminados», en el segundo escenario, la «onda expansiva» del resultado no tiene un itinerario prefijado.

Resulta interesante la denominación de «referéndum políticamente vinculante». Autores como Bicquelet y Addison ${ }^{28}$ explican que en determinados contextos, y es el caso de los urdidos acerca de la participación o

revision; unity of hierarchical level: it is desirable that the same question should not simultaneously apply to legislation of different hierarchical levels, ...». Vid. http://www.venice.coe.int/ webforms/documents/default.aspx?pdffile $=c d l$-ad(2007)008-e.

28 A. BicQuelet y H. AdDISON, «Are Discretionary Referendums on EU Integration 
permanencia en la Unión Europea, la estrategia de los gobiernos y el tipo/ modo de consulta convierte a estas en vinculantes desde la perspectiva política. Ya no puede, a pesar de la inexistencia de normas jurídicas precisas en tal sentido, ser desatendida la proclama ciudadana que está cifrada en el resultado de ese referéndum concreto. Esa soberanía inalienable e imprescriptible perfilada por Bodin en sus escritos sobre la república no puede silenciarse, no puede ignorarse.

\section{VII. ¿QUIÉN TIENE O DEBERÍA TENER LA COMPETENCIA PARA CONVOCAR UN REFERÉNDUM?}

Este asunto es decisivo y así lo ponen de relieve los distintos países democráticos del siglo XXI. ¿Quién tiene la competencia para plantear y convocar una consulta de esta naturaleza y quién debería tenerla? Es necesario establecer unas reglas claras que proporcionen «seguridad y legitimidad» a una convocatoria de referéndum.

En España la normativa es clara (art. 2): «La autorización para la convocatoria de consultas populares por vía de referéndum en cualquiera de sus modalidades es competencia exclusiva del Estado. La autorización será acordada por el Gobierno, a propuesta de su presidente, salvo en el caso en que esté reservada por la Constitución al Congreso de los Diputados. Corresponde al rey convocar a referéndum mediante real decreto acordado en Consejo de Ministros y refrendado por su presidente».

Pero en un gran número de países la iniciativa de referéndum puede partir de la ciudadanía recogiendo un número de firmas determinado que demuestren el suficiente apoyo popular. En los Países Bajos son necesarias 10.000 firmas para iniciar un proceso de consulta y 300.000 para poder celebrarlo (Consultative Referendum Act 2014). En Italia se requieren 500.000 firmas (arts. 75 y 138 de la Constitución italiana). También determinadas mayorías parlamentarias pueden lanzar y celebrar un referéndum (Austria, España o Italia). En todo caso, el mundo de los datos y de las diversas opciones confluye en la pregunta, ¿debe ampliarse o restringirse la posibilidad de empoderar o ampliar la posibilidad de sujetos legitimados para solicitar y autorizar la celebración de un referéndum? Evidentemente serán decisivos otros elementos tales como: si estamos ante modelos

Becoming Politically Obligatory? The Cases of France and UK», Parliamentary Affairs: a Journal of representative politics, vol. 71, núm. 2 (2018), pp. 219-241. 
constitucionales escritos o «unwritten» (Reino Unido o Nueva Zelanda), el tipo de mayorías o firmas requeridas para solicitar/celebrar un referéndum, el marco político/formal en el que se propone, si el tipo de referéndum es obligatorio o no, etcétera.

Y siendo este asunto de gran complejidad, solo queremos poner de relieve, en línea con las pretensiones de estas páginas, algunas reflexiones que sigan contribuyendo al debate. El marco que debe dilucidar, frente a las referidas preguntas, las respuestas, enfrenta o pone en conflicto diversos tipos de «legitimidades»: si el referéndum es sobre una ley en vigor, sobre una reforma constitucional, sobre una ley de ratificación de un tratado internacional, etcétera.

Frente a la «soberanía parlamentaria» se expresa la soberanía popular que alumbró aquella en su momento. Este sería un debate en bucle; así lo protagoniza la actualidad británica tras el referéndum del Brexit en 2016, en un modelo de constitución no escrita y con unas reglas indefinidas y más bien «laxas» sobre la figura del referéndum.

Leeman explica que si los ciudadanos pueden potencialmente «forzar» a votar ciertas cuestiones políticas a los gobernantes, estos de algún modo se sienten obligados a perseguir y procurar políticas más próximas y válidas para los ciudadanos ${ }^{29}$. En Estados Unidos también se pone de relieve que en aquellos Estados en los que los ciudadanos tienen la titularidad y competencia de convocar referéndums, las políticas del Gobierno se perciben y evalúan como más próximas a sus necesidades $^{30}$. Pero también debe aceptarse que esta tendencia a la «ampliación» de procesos de referéndum tiene riesgos inherentes de presiones de minorías, desgaste del gobierno, campañas inapropiadas o «desinformadoras», lesivas, costes económicos estériles y gravosos o «perversión» de la participación.

${ }^{29}$ L. LeEman, «Is Direct Democracy Effective? Yes, if it is Citizens who Start the Process», Democratic Audit, 22 de junio de 2018, disponible en http://www.democraticaudit. com/2016/06/22/is-direct-democracy-effective-yes-if-it-is-citizens-who-start-the-process/.

${ }^{30}$ L. JacobsmeIER, «Evaluating Policy Representation with Dynamic MRP Estimates: Direct Democracy and Same-Sex Relationship Polices in the United States», State Politics and Policy Quarterly, vol. 17, núm. 4 (2017), pp. 450 y ss. 


\section{CONCLUSIONES}

1. El referéndum es una herramienta de participación de la soberanía popular. Cualquier herramienta debe diseñarse al servicio del objetivo para el que se crea. La actualidad de los últimos años pone de relieve un incremento de los referéndums sobre cuestiones políticas básicas o sobre aspectos constitucionales esenciales. La tendencia de este siglo XXI, a nuestro juicio negativa, revela que se han enfocado y dirigido este tipo de procesos no tanto de acuerdo con principios esenciales democráticos o pautas constitucionales básicas en cualquier democracia consolidada, sino atendiendo a urgencias, coyunturas y pragmatismos políticos.

2. El referéndum, en tanto que mecanismo de participación democrática, debe permitir y propiciar deliberación, negociación, compromiso, responsabilidad, seguridad, claridad, legitimidad, transparencia, etc. Es, sin duda, mucho más que un simple voto, una respuesta rápida o una simple votación. Ahora bien, no siempre es la mejor opción frente a un proceso de decisión política: la acción del gobierno, el rol del parlamento y sus funciones, la negociación y debate entre partidos políticos, entre otros, no pueden ser alter egos del referéndum. Tales consultas requieren un previum claro y seguro, y no pueden nacer de la improvisación o la mera «alternancia» de la participación política.

3. La forma del referéndum es muy importante. Cualquier herramienta es «limitada» y no apropiada para todo tipo de consultas. El contenido del referéndum debe ser objeto de análisis y regulación. No parece posible utilizar esta herramienta para cualquier tipo de participación, so pena de lesionar la esencia de la democracia y de tergiversar la legitimidad. La realidad pone de relieve que el referéndum puede fortalecer y debilitar la democracia, de ahí la importancia de un buen uso y diseño del mismo.

4. El momento o temporalidad del referéndum es muy relevante. La legislación o normativa de regulación a este respecto debe ser segura y clara. La actualidad demuestra que los referéndums prelegislativos son «complejos» y debilitadores de la fortaleza o esencia de la democracia, pero la «oportunidad» también debe ser sopesada, descartando escenarios o circunstancias que podrían falsear o alterar el devenir justo y claro de este mecanismo.

5. Por todo ello, los efectos de los potenciales resultados del referéndum deben estar claros y «prefijados». Efectos que van más allá de resulta- 
dos vinculantes o no vinculantes, porque existen los denominados «efectos de vinculación política» inherentes a la naturaleza de la soberanía popular consultada.

Virgala Foruría expone de manera brillante un escenario en el que podemos ver claramente la enorme importancia que ha adquirido en nuestros días el referéndum como «mecanismo constitucional» ${ }^{31}$. Ello sin duda constituye un reclamo para su permanente reflexión y actualización.

31 E. VIRgala ForuRIa, La Constitución británica en el siglo XXI. Soberanía parlamentaria, constitucionalismo common law y leyes constitucionales, Madrid, Marcial Pons, 2018, p. 18: «La Constitución británica ha vivido un proceso de cambio continuo que probablemente solo pueda darse en una Constitución no codificada de este tipo, desde el big bang que supuso el Gobierno laborista de Blair (1997-2007) con la HRA de 1998, el proceso de devolución territorial de ese mismo año a Escocia e Irlanda del Norte, luego extendido a Gales, la reforma de los Lores de 1999, la Freedom of Information Act de 2000, la reforma judicial de 2005 y la puesta en marcha del TS-RU en 2009; y el Gobierno de coalición conservador-liberaldemócrata de Cameron (2010-2015) con la legislatura fija de cinco años en la Fixed-Term Parliaments Act de 2011, el referéndum sobre la independencia de Escocia en 2014 y, finalmente, el Gobierno solo conservador de Cameron (2015-2016) con el EVEL en 2015 y los distintos avatares de su relación con la UE hasta el referéndum del Brexit de 2016. A ello se añade la utilización frecuente del referéndum como mecanismo constitucional». 